\title{
ON THE ZEROS OF EXPONENTIAL SUMS AND INTEGRALS*
}

BY R. E. LANGER

1. Introduction. It is of frequent occurrence in problems of both pure and applied mathematics that certain values sought may be specified and must be determined as the roots of a transcendental equation. In particular, the equation may be of the class in which the unknown is involved only through the medium of exponential or trigonometric functions, with coefficients which are power functions or essentially such. As simple examples one has such equations as

$$
\begin{gathered}
\tan z-z=0, e^{z}+z^{2} e^{3 z}+(1-z)=0, \\
\sin z+z^{3} \tan 5 z=0, e^{i z}-2 z^{5}=0 .
\end{gathered}
$$

A determination of the distribution of roots of such an equation may, therefore, be of interest and importance. Inasmuch as this problem and certain of its generalizations have been the subjects of recent and successful investigations I have presumed that an outline of methods and attainments associated with it might not be unsuitable for presentation at this meeting of the Society.

The relation between the trigonometric functions of $z$ and the function $e^{i z}$ evidently makes possible the expression of each of the functions involved in the equations above in the form

$$
\Phi(z)=\sum_{j=0}^{n} A_{j}(z) e^{c_{j} z} .
$$

This type of function we shall refer to as an exponential sum. It is to be the subject of Part I of the discussion to follow. If the values $A_{j}(z)$ and $c_{j}$ are constants, and the $c_{j}$ are real, the function (1) can be expressed with the use of an appropriate constant $z_{0}$ and a suitable step-function $\phi(t)$ in the form

* An address delivered at the meeting of the Society at Columbia, Missouri, November 29, 1930, by invitation of the program committee. 


$$
\Phi\left(z-z_{0}\right)=z \int_{c_{0}}^{c_{n}} \phi(t) e^{t z} d t .
$$

The integral of the type here involved with less specialized function $\phi(t)$ represents, therefore, a generalization of certain sums of type (1). Such integrals include a number of standard functions of importance, among which the Bessel functions may be specifically mentioned. They will be considered briefly in part II of this discussion.

Returning to the consideration of the sum (1) we shall understand that the quantities $c_{j}$, to be called the exponents, are constants (real or complex). The functions $A_{j}(z)$, to be called the coefficients, will be characterized as the discussion progresses by various sets of hypotheses. It will be my general plan to pass in the considerations from forms of less to forms of greater generality, and to indicate at each stage the results and their relation with those previously established.

\section{Part I. The Exponential Sum}

2. Constant Coefficients and Real Commensurable Exponents. When the exponents $c_{j}$ in a sum of the form (1) are real, it is a matter merely of the arrangement of terms to suppose that these exponents occur in the order of increasing algebraic magnitude. With this arrangement it is then permissible without loss of generality to assume that the first exponent $c_{0}$ is zero, inasmuch as the removal of any exponential factor can in no way affect either the number or distribution of the zeros of the function. These adjustments will be assumed to have been made. Theoretically the simplest type of sum is one, then, in which the problem of the distribution of zeros is essentially an algebraic one, and this occurs in particular when the coefficients are constants $\left(A_{j}(z) \equiv a_{j}\right)$, and the exponents are commensurable, that is, $c_{j}=\alpha p_{j}, j=1,2, \cdots, n$, with some real constant $\alpha$ and integral values $p_{j}$. The sum is then of the form

$$
\Phi(z)=\sum_{j=0}^{n} a_{j}\left(e^{\alpha z}\right)^{p_{j}}, p_{0}=0
$$

which is a polynomial of degree $p_{n}$ in the quantity $e^{\alpha z}$. If this polynomial admits as zeros the values $\xi_{1}, \xi_{2}, \cdots, \xi_{p_{n}}$, the func- 
tion (3) vanishes for such values of $z$, and for only such, as satisfy a relation

$$
e^{\alpha z}=\xi_{j} .
$$

The zeros of $\Phi(z)$ are, therefore, given by the formula

$$
z=\frac{1}{\alpha}\left\{2 m \pi i+\log \xi_{j}\right\}, \quad \begin{array}{r}
\left(j=1,2, \cdots, p_{n}\right), \\
(m=0, \pm 1, \pm 2, \cdots) .
\end{array}
$$

They are thus seen to be denumerably infinite in number and to be distributed in the complex $z$ plane at regular intervals of length $2 \pi / \alpha$ along $p_{n}$ lines (not necessarily distinct) which are normal to the axis of reals.

If the explicit solution of the polynomial equation involved is feasible, the determination of the zeros of $\Phi(z)$ is completed by the formula (4). In the alternative case the following further considerations will not be futile. It is clear from the result (4), that with any specifically given function $\Phi(z)$, the choice of a constant $K$ is possible so that the zeros of $\Phi(z)$ all lie within the rectilinear strip of the $z$ plane given by the relation

$$
|x|<K, \quad(z=x+i y) .
$$

Any line $y=y_{1}$, parallel to the axis of reals, cuts this strip, and on such a line it is found by direct computation that

$$
I\left\{\frac{1}{a_{0}} \Phi(z)\right\}=\sum_{j=1}^{n} b_{j}\left(y_{1}\right)\left(e^{\alpha x}\right)^{p_{j}}
$$

the coefficients $b_{j}$ being real constants with values depending upon $y_{1}$. The expression (6) is a polynomial in the positive quantity $e^{\alpha x}$. As such it can vanish, by Descartes's rule, at most as many times as there are changes of sign in the sequence of values $b_{j}$. This cannot exceed $(n-1)$, since the number of terms in (6) is $n$.

Let $R$ designate the rectangular region cut from the strip (5) by two lines $y=y_{1}$, and $y=y_{2}$, chosen so that on neither of them there lies a zero of $\Phi(z)$. (There will evidently be such lines through any interval of the $y$ axis, however small.) Then as $z$ traces either of these lines the value arg $\Phi(z)$ can change (increase or decrease) by at most $n \pi$ since $I\left\{\left(1 / a_{0}\right) \Phi(z)\right\}$ vanishes no oftener than $(n-1)$ times. As $z$ traces the side $x=-K$ of 
the rectangle $R$ the value of the sum $\Phi(z)$ remains arbitrarily near that of its first term $a_{0}$ (provided $K$ has been chosen sufficiently large), and the change in $\arg \Phi(z)$ accordingly remains arbitrarily near to 0 . Lastly as $z$ traces the side $x=K$ the change in $\arg \Phi(z)$ is an increase in value arbitrarily near to $c_{n}\left(y_{2}-y_{1}\right)$, since $\Phi(z)$ on this side of the rectangle is approximated with an arbitrary degree of accuracy by its last term $a_{n} e^{c_{n} z}$. From this evaluation of the extreme possible changes in arg $\Phi(z)$ as $z$ traces the boundary of the rectangle it must be concluded that the number $n(R)$ of zeros of $\Phi(z)$ within the rectangle is subject to the bounds

$$
-n+\frac{c_{n}}{2 \pi}\left(y_{2}-y_{1}\right) \leqq n(R) \leqq n+\frac{c_{n}}{2 \pi}\left(y_{2}-y_{1}\right) .
$$

This evidently limits, both above and below, the density of zeros in any portion of the strip (5). In particular it shows that no sum of the form (3) with $(n+1)$ terms can have a zero of multiplicity greater than $n$.

THEOREM 1. If in the exponential sum (1) the coefficients are constants and the exponents are real and commensurable, then the distribution of zeros is given explicitly by the formula (4). In this distribution the number of zeros which lie between two lines $y=y_{1}$, and $y=y_{2}$, is restricted by the relation (7).

The hypothesis of commensurability of the exponents is evidently a restrictive one. Yet many functions $\Phi(z)$ of importance and interest are included by it. It will be observed, for instance, that the hypothesis is vacuous whenever the sum involves only two terms, and the results obtained apply, therefore, to all such sums. Similarly the familiar trigonometric sums

$$
\begin{gathered}
\Phi_{c}(z)=\sum_{j=0}^{n} a_{j} \cos j z, \\
\Phi_{s}(z)=\sum_{j=1}^{n} a_{j} \sin j z
\end{gathered}
$$

are included, as is in fact any linear combinaton of trigonometric functions of integral multiples of $z$ ( $i z$ naturally replacing $z$ ). While the problem is in this case theoretically simple, the interrelation of algebraic and transcendental features may be 
of distinct interest. The following theorem, due to Pólya, ${ }^{*}$ is at once illustrative of this point and useful for later reference.

ThEOREM 2. If the coefficients $a_{j}$ are real and the zeros of the polynomial

$$
P(\xi)=\sum_{j=0}^{n} a_{j} \xi^{j}
$$

all lie within the unit circle about $\xi=0$, then the zeros of the corresponding trigonometric sums (8) are all real and simple. Each of these sums has precisely $2 n$ zeros on the interval $0 \leqq z<2 \pi$ and the zeros of either sum alternate with those of the other. (By a theorem of Kakeya the hypothesis is fulfilled if $0 \leqq a_{0}<a_{1}<\cdots<a_{n}$.)

The proof is exceedingly simple. Under the hypothesis made arg $P(\xi)$ increases by $2 n \pi$ as $\xi$ traces the unit circle, that is, arg $P\left(e^{i z}\right)$ increases by that amount as $z$ traces the axis of reals from 0 to $2 \pi$. But when $z$ is real the sums (8) are respectively the real and imaginary parts of $P\left(e^{i z}\right)$. Hence these sums vanish in alternation, each $2 n$ times on the interval $0 \leqq z<2 \pi$. That the zeros are thus all accounted for follows from the fact that each of the sums (8) may be expressed as a polynomial of degree $2 n$ in $e^{i z}$.

3. Constant Coefficients and General Real Exponents. When the exponents are not commensurable the determination of the distribution of zeros of $\Phi(z)$ is not in general of an algebraic character. Independent discussions of the problem in this case have been given by C. E. Wilder ${ }^{2}$ and by J. D. Tamarkin. ${ }^{3}$ Inasmuch as the subject has to some extent been anticipated by the discussion of the preceding section a slight amplification of the reasoning there used will suffice here for the derivation of such results as have been obtained.

The sum $\Phi(z)$ for the case in hand is expressed by the formula

$$
\Phi(z)=\sum_{j=0}^{n} a_{j} e^{c_{j z}}, \quad c_{0}=0 .
$$

Given such a function it is easily seen that with $K$ chosen as a constant sufficiently large the zeros of $\Phi(z)$ all lie within a strip of the $z$ plane determined by a relation (5). This may be de-

* See list of references at the end of this paper. 
duced from the dominance of the first term of the sum (10) over all remaining terms for values of $z$ satisfying the condition $x<-K$, and the similar dominance of the last term of the sum when $x>K$. The change in $\arg \Phi(z)$ as $z$ traces any portion of the lines $x=-K$ or $x=K$ may be computed precisely as in the case of the preceding section and the establishment of the relation (7) depends only on the consideration of the quantity

$$
I\left\{\frac{1}{a_{0}} \Phi(z)\right\}=\sum_{j=1}^{n} b_{j}(y) e^{c j x},
$$

on a line for which $y$ is constant. Since the expression (11) is now not a polynomial Descartes's rule may not be invoked. It is, however, easily proved that in this case also the expressions can vanish at most as many times as there are changes in sign in the sequence of coefficients (see Pólya and Szegö, Aufgaben und Lehrsätze aus der Analysis, vol. 2, p. 49) and hence the relation (7) is valid as before for any rectangle $R$ cut from the strip (5) by a pair of lines $y=y_{1}$ and $y=y_{2}$ on neither of which $|\Phi(z)|$ is zero. The existence of such lines through any interval of the $y$ axis follows, of course, from the regularity of the function $\Phi(z)$.

It is a fact of considerable importance in many considerations that in the case of an exponential sum the value $|\Phi(z)|$ is uniformly bounded from zero when the variable $z$ is uniformly bounded from the zeros of $\Phi(z)$. This means that given a sufficiently small positive $\delta$ there exists a constant $H$ depending only on $\delta$ and such that

$$
|\Phi(z)|>H, \text { for }\left|z-z_{m}\right|>\delta,
$$

where $z_{m}$ designates the set of zeros of $\Phi(z)$. The fact is obvious when consideration is restricted to any finite portion of the $z$ plane. In the case of commensurable exponents it then follows for the entire strip (5) and hence for the entire $z$ plane because of the periodicity of the function $\Phi(z)$. The fact may also be established (see Wilder ${ }^{2}$ or Tamarkin ${ }^{3}$ ), though less simply, in the general case.

THEOREM 3. If in the exponential sum (10) the coefficients are constants and the exponents are real, then the zeros of the sum all lie within a strip (5), and in any portion of this strip the number 
of zeros is limited by the relation (7). When $z$ is uniformly bounded from the zeros of $\Phi(z)$, then $|\Phi(z)|$ is uniformly bounded from zero.

It may not be devoid of interest to remark that the distance between distinct zeros of $\Phi(z)$ admits of no positive lower bound in the general case of incommensurable exponents. This is shown by the simple and explicit example $\Phi(z)=\left(1-e^{\alpha z}\right)\left(1-e^{\beta z}\right)$, in which $\alpha / \beta$ is irrational. The formula (4) shows that when the exponents are commensurable a minimum distance between zeros does exist.

4. Coefficients Asymptotically Constant. In many considerations associated with the distribution of zeros of a function of type (1) the interest centers directly only upon the character of this distribution in the region of the $z$ plane remote from the origin, that is, in the region $|z|>M$, where $M$ is a constant arbitrarily large. When such is the case it is natural that the hypothesis of the constancy of the coefficients may be replaced without essential loss by a less stringent one to the effect that the coefficients be sufficiently like constants when $|z|$ is large. To facilitate the formulation of such hypotheses we shall agree upon the use of symbolism as follows. In a region $R$ of the $z$ plane including the point $z=\infty$ a function will be called an epsilon function provided that it is analytic in every finite portion of $R$ and that it approaches zero uniformly in $R$ as $|z| \rightarrow \infty$. To indicate such a function we shall use the generic symbol $\epsilon(z)$.

Let it be supposed now either that the coefficients $A_{j}(z)$ in the sum (1) are single valued and of the form

$$
A_{j}(z)=a_{j}+\epsilon(z),
$$

in the region $|z|>M$, or that they are multiple valued but such that in the region $|z|>M,-\pi<\arg z \leqq \pi$, their various branches are each of the form (13). In the latter case the several determinations of the coefficients correspond to distinct branches of the sum $\Phi(z)$, and the considerations which follow apply directly to any one of these branches. The discussion may be completed by a similar consideration of each individual branch. The form assumed for the sum (1) is, therefore,

$$
\Phi(z)=\sum_{j=0}^{n}\left\{a_{j}+\epsilon(z)\right\} e^{c j z}, \quad a_{0} a_{n} \neq 0 .
$$


As already indicated it will be made a part of the hypothesis that neither of the two constants $a_{0}, a_{n}$ is zero. It will be shown that the zeros of the sum (14) are asymptotically represented by those of the related sum

$$
\Phi_{1}(z)=\sum_{j=0}^{n} a_{j} e^{c j z}
$$

which is of the type considered in $\$ \S 2$ and 3 .

It may be shown to begin with, and by precisely the reasoning employed in the analogous consideration of $\S 3$, that there can be no zeros of the sum (14) outside a strip of the form (5) when $|z|>M$. Within this strip, on the other hand, each exponential is bounded and hence the function (14) is evidently expressible in the form

$$
\Phi(z)=\Phi_{1}(z)+\epsilon(z), \quad|x|<K,|z|>M .
$$

Now in virtue of the distribution of zeros of $\Phi_{1}(z)$, as determined in the preceding section, these zeros may be enclosed in groups of $n$ or less by a succession of contours $\gamma_{r}$ such that no point of such a contour lies within a distance less than $\delta$ from a zero of $\Phi_{1}(z)$, and each of the regions enclosed is of major diameter $n \delta$. For values of $z$ not within such a contour, $\left|\Phi_{1}(z)\right|$ is bounded uniformly from zero, as stated in Theorem 3, and hence for such values of $z$,

$$
\Phi(z)=\Phi_{1}(z)\{1+\epsilon(z)\} .
$$

From this relation it follows that when $|z|>M, \Phi(z)$ has within each contour $\gamma_{r}$ precisely as many zeros as $\Phi_{1}(z)$, and that all zeros of $\Phi(z)$ are thereby accounted for.

THEOREM 4. If the function $\Phi(z)$ (or a determination of $i t$ ) is of the form (14), then in the region $|z|>M$ the distribution of zeros of $\Phi(z)$ (or of the branch of $\Phi(z)$ in question) may be described as in Theorem 3. The zeros are asymptotically represented by those of the related sum (15).

5. Coefficients which are Asymptotically Power Functions. It will be supposed now that in the form (1) the coefficients $A_{j}(z)$, or chosen branches of them, are of the form

$$
A_{j}(z)=z^{\nu_{j}}\left\{a_{j}+\epsilon(z)\right\}, \quad a_{0} a_{n} \neq 0,
$$


for $z$ in the region $|z|>M,-\pi<\arg z \leqq \pi$. The values $\nu_{j}$ are taken to be any real constants positive, negative or zero.

The sums with coefficients of type (16) evidently include as a sub-class those with coefficients which are polynomials in $\boldsymbol{z}$. A discussion of the latter was given first by $\mathrm{Poblya}^{6}$ and Schwengler. ${ }^{7}$ Their method, which is largely geometric, is in many respects convenient and illuminating and will be utilized in the following discussion whenever it is of advantage.

Without further convention the values $\nu_{j}$ (except for $j=0$, and $j=n$ ) are not fixed beyond ambiguity by the form (16), inasmuch as an increase in $\nu_{j}$ may be compensated for by the choice of $a_{j}$ as zero. It will be assumed, therefore, for the sake of definiteness that whenever the form of the coefficient $A_{j}(z)$ permits, the value $\nu_{j}$ will be chosen so that the corresponding constant $a_{j}$ is not zero. The terms of the sum for which this is possible will be called ordinary terms. In the exceptional nonordinary terms the value of $\nu_{j}$ must remain unspecified. Under the form (16) the constant $a_{j}$ for every such term will be zero. Thus, by way of example, if $A_{j}(z)=\log z$, then the form (16) implies $a_{j}=0$, with $\nu_{j}>0$.

As a preliminary to the discussion of the general case under (16) it will be convenient to consider that in which all terms are ordinary terms, and in which the constants $\nu_{j}$ are proportional to the exponents $c_{j}$.

6. The Values $\nu_{j}$ and $c_{j}$ Proportional. If the real constant $\beta$ is defined by the relation

$$
\nu_{j}=\beta c_{j}, \quad(j=1,2, \cdots, n),
$$

the formula

$$
\zeta=z+\beta \log z
$$

defines a single-valued analytic map of the portion of the $z$ plane to which $z$ was restricted above upon a complex $\zeta$ plane, the point $z=\infty$ corresponding to $\zeta=\infty$. It follows that any epsilon function of $z$ is also such a function of $\zeta$, and it is evident, therefore, that under the relation (17)

$$
\Phi(z) \equiv \sum_{j=0}^{n}\left\{a_{j}+\epsilon(\zeta)\right\} e^{c_{j} \zeta}, \quad a_{0} a_{n} \neq 0
$$


This, however, as a function of $\zeta$ is precisely of the form (14), and in the $\zeta$ plane the zeros are accordingly distributed as described in Theorem 4 . It is necessary only to translate this result into terms of the variable $z$ and this can be readily done. Thus if $\zeta=\xi+i \eta$ the components of the relation (17) are

$$
\begin{aligned}
& \xi=x+\beta \log |z|, \\
& \eta=y+\beta \arg z .
\end{aligned}
$$

The rectilinear strip $|\xi|<K$ corresponds, therefore, to the curvilinear strip bounded by the logarithmic curves

$$
x+\beta \log |z|= \pm K .
$$

This strip which is asymptotically of constant width $2 K$, and whose bounding curves approach parallelism with the axis of imaginaries, contains all the zeros of $\Phi(z)$ in the region $|z|>M$. Since for $z$ in the strip (18) the value arg $z$ approaches either $\pi / 2$ or $-\pi / 2$ according as the upper or lower half plane is considered, it follows that any line $\eta=\eta_{1}$ on which $\eta$ is constant maps asymptotically upon the line $y=\eta_{1} \pm \beta \pi / 2$, on which $y$ is constant. Moreover, the distance between two such lines both in the upper or both in the lower half plane is asymptotically preserved.

THEOREM 5. If in the exponential sum (1) the coefficients are of the form (16) with values $\nu_{j}$ proportional to the exponents $c_{j}$, and all terms are ordinary terms, then the zeros of the sum are asymptotically located within a logarithmic curvilinear strip bounded by curves of the form (18), and the number of zeros lying between any two lines parallel to the axis of reals is asymptotically subject to the relations (7).

7. General Real Values $\nu_{j}$. With each ordinary term of the sum

$$
\Phi(z)=\sum_{j=0}^{n} z^{v_{j}}\left\{a_{j}+\epsilon(z)\right\} e^{c j z},
$$

there can be associated in the $z$ plane the corresponding point $P_{j}$ with Cartesian coordinates $\left(c_{j}, \nu_{j}\right)$. These points determine the broken (or straight) line $L$, which (i) joins $P_{0}$ with $P_{n}$, (ii) has vertices only at points of the set $P_{j}$, (iii) is convex upward (or straight), and (iv) is such that no points of the set lie above it. Let $L_{r}$ designate the $r$ th segment of this broken line $L$, the 
order being from left to right, and let the scheme of single subscript notation employed in (19) and in designating the set of points $P_{j}$ be replaced by one in which the points of the set lying on the segment $L_{r}$ are denoted in order by $P_{r h}, \quad(h=1$, $\left.2, \cdots, n_{r}\right)$.

If the sum (19) contains exceptional terms it must be made a matter of hypothesis that for every such term a choice of the value $\nu_{j}$ is possible under the form (16) such that the corresponding point $\left(c_{j}, \nu_{j}\right)$ lies below the broken line $L$. All points of the set located below the line $L$ may then be designated in any order by $P_{0 h},\left(h=1,2, \cdots, n_{0}\right)$, similar double subscripts being assigned to the corresponding terms in (19).

Consider now any intermediate segment $L_{r}$ of the line $L$, and let its slope be $m_{r}$, the slope of the preceding segment being $m_{r-1}$. Then as the real parameter $k$ varies over the range

$$
m_{r-1}-\epsilon \geqq k>m_{r}-\epsilon,
$$

with $\epsilon$ positive and sufficiently small but otherwise arbitrary, the curve

$$
x=-k \log |z|
$$

sweeps out the region of the $z$ plane bounded by the curves

$$
\begin{aligned}
& x=-\left(m_{r-1}-\epsilon\right) \log |z|, \\
& x=-\left(m_{r}-\epsilon\right) \log |z| .
\end{aligned}
$$

The figure is symmetrical in the upper and lower half planes. On any curve $(20)$ it is obvious that

$$
\left|z^{\nu_{l s}} e^{c_{l_{s}}}\right|=|z|^{\nu_{l s}-k c_{l s}} \text {, }
$$

and hence the relative magnitude of the terms in (19) for $z$ on this curve depends upon the corresponding values $\left(\nu_{l_{s}}-k c_{l_{s}}\right)$. These, however, admit of ready geometrical interpretation. They are, namely, the $y$ intercepts of the lines having the slope $k$ and passing through the respective points $P_{l s}$. In virtue of this fact and the restriction upon the range of $k$ it is readily seen that for every $l \neq r$ the inequality

$$
\left(\nu_{l s}-k c_{l_{s}}\right)<\left(\nu_{r 1}-k c_{r 1}\right)
$$

obtains. It follows, therefore, that the form of expression

$$
z^{\nu_{l s}} e^{c_{8} z}=z^{\nu_{1}} e_{r_{1}}^{c_{1}} \in(z), \quad l \neq r,
$$


is valid for $z$ on any curve (20), and hence that the sum (19) can be writt $2 n$ in the form

$$
\Phi(z)=\sum_{h=1}^{n_{r}} z^{v_{r h}}\left\{a_{r h}+\epsilon(z)\right\} e^{c_{r h} z},
$$

for all $z$ of the region bounded by the curves (21). Upon removal of a factor $z^{v_{r_{1}}} e^{c_{1} z}$, however, the sum (22) takes the form considered in $\$ 6$, since the constants $\left(\nu_{r h}-\nu_{r 1}\right)$ are proportional to the exponents $\left(c_{r h}-c_{r 1}\right)$, the constant of proportionality being $m_{r}$, the slope of the segment $L_{r}$. It may be concluded, therefore, by Theorem 5 , that the zeros of $\Phi(z)$ in the region (21) are asymptotically confined to the logarithmic strip bounded by the curves

$$
x+m_{r} \log |z|= \pm K,
$$

and that the number of zeros in this strip and between two lines $y=y_{1}$ and $y=y_{2}$ is restricted by the relation obtained from (7) by replacing $n$ and $c_{r}$ by $n_{r}$ and $\left(c_{r, n_{r}}-c_{r_{1}}\right)$.

The geometric picture is completed if it is observed that in the upper half of the plane the central line of the strip (23), that is, the curve

$$
x+m_{r} \log |z|=0,
$$

passes through the point $(0,1)$ with slope $-1 / m_{r}$, namely in the direction perpendicular to that of the associated segment $L_{r}$. There is a strip of the form (23) associated with each segment of the broken line $L$ (obvious simple modifications of the discussion being necessary in the case of an end segment), and the zeros of $\Phi(z)$ in the remote part of the $z$ plane are all contained within these strips.

THEOREM 6. If $\Phi(z)$ is an exponential sum with coefficients of the form (16) (the $\nu_{j}$ for exceptional terms satisfying the hypothesis of the text), then the zeros of the sum are asymptotically confined to a finite number of logarithmic strips (23), the number of zeros in any strip between two lines parallel to the axis of reals being asymptotically subject to a relation similar to (7).

8. Collinear Complex Exponents. The hypotheses which have been made in the various cases of the sum (1) hitherto considered have been concerned primarily with the structure of the 
coefficient functions $A_{j}(z)$. The exponents have been assumed throughout to be real. It will be shown now that this assumption is dispensable, and that the distribution of zeros of the sum having complex exponents is also determinable. Preliminary to the general discussion the considerations of this section will be given to the case in which the values $c_{j}$ viewed as points in the complex plane are collinear.

Let $l$ designate the line on which the points $c_{j}$ are located, and let the subscripts be assigned to these points in the order of their location on $l$. Then if $\theta$ designates the inclination angle of the line $l$ with respect to the axis of reals (the positive sense of $l$ being from $c_{0}$ to $c_{n}$ ), it follows that the relations

$$
c_{j}=c_{0}+\gamma_{j} e^{i \theta}, \quad(j=0,1,2, \cdots, n),
$$

are satisfied by a set of real values $0=\gamma_{0}<\gamma_{1}<\cdots<\gamma_{n}$. In terms of the variable $\zeta$ defined by the relation

$$
\zeta=z e^{i \theta},
$$

the sum (1), therefore, takes the form

$$
\Phi=e^{c_{0} z} \sum_{j=0}^{n} B_{j}(\zeta) e^{\gamma_{j} \zeta}
$$

where $B_{j}(\zeta) \equiv A_{j}(z)$ under the substitution (25). The sum in (26) is one in which the exponents $\gamma_{j}$ are real, and the coefficients $B_{j}(\zeta)$ have the essential structural characteristics of $A_{j}(z)$. The distribution of zeros of the sum $\Phi$ in the $\zeta$ plane is, therefore, as described in the theorems of the preceding sections, that is, the zeros are asymptotically confined to one or more strips which are parallel or approach parallelism with the axis $\arg \zeta= \pm \pi / 2$. Since the transformation (25) is a mere rotation of the plane, it may be concluded that in the $z$ plane the zeros of the sum are confined for $|z|>M$ to a similar set of strips parallel or approaching parallelism with a direction arg $z=-\theta$ $\pm \pi / 2$. These directions, it will be noted, are those perpendicular to the line in the complex plane which contains the points $\bar{c}_{j}$, the complex conjugates of the exponents $c_{j}$.

THEOREM 7. If the exponents $c_{j}$ in the exponential sum (1) are collinear complex constants, the distribution of zeros of $\Phi(z)$ is obtainable from the theorem previously enunciated by substituting 
in the role of the axis of reals the line containing the points $\bar{c}_{j}$ conjugate to the exponents $c_{j}$.

9. General Complex Constants. In the most general type of exponential sum to which consideration will here be given the exponents may be any set of complex constants. This case is now mature for discussion. While treatments of it by purely analytic methods are available ${ }^{2,3,4,5}$ and effective, a geometric method $^{6}$ will again be resorted to as convenient for the purpose at hand. Let it be supposed that the values $\bar{c}_{j}$ (conjugate to the exponents) have been plotted in the complex plane, and let the points of the set so represented be surrounded by the polygon $P$ which (i), is convex, (ii), has vertices only at points of the set, and (iii), includes all points of the set either in its interior or on its perimeter.

Let the sides of the polygon $P$ taken in counterclockwise succession with any one as the initial one be designated by $l_{r}$, $r=1,2, \cdots, q$, and with this done let the points of the set $\bar{c}_{j}$ which lie on the side $l_{r}$ be redesignated by $\bar{c}_{r h}, h=1,2, \cdots, h_{r}$, the succession being again in the counterclockwise sense around the polygon $P$. The points $\bar{c}_{j}$ lying within the polygon may in conclusion be denoted (in any order) by $\bar{c}_{0 h}, h=1,2, \cdots, h_{0}$.

If $\omega_{r}$ represents the inclination angle of the outer normal to the side $l_{r}$, the relation

$$
\omega_{r-1}+\epsilon \leqq \tau<\omega_{r}+\epsilon,
$$

with $\epsilon$ sufficiently small and positive but otherwise arbitrary, restricts the real parameter $\tau$. As $\tau$ varies over the range permitted, the line

$$
\arg z=\tau
$$

sweeps out in the $z$ plane a sector described by the relation

$$
\omega_{r-1}+\epsilon \leqq \arg z<\omega_{r}+\epsilon .
$$

Such a sector is thus associated with each side of the polygon, and the set of these sectors completely fills out the complex plane. It is proposed to consider the sum $\Phi(z)$ within the typical sector (28). The extension of the results to the entire plane will then be immediate. 
When $z$ lies on a line (27) it is readily computed that

$$
\left|z^{\nu} k c_{s k} z\right|=e^{|z|\left\{\alpha_{s k} \cos \tau+\beta_{s k} \sin \tau+\nu_{s k} \log |z| /|z|\right\}}
$$

where $\bar{c}_{s k}=\alpha_{s k}+i \beta_{s k}$. It follows from this that the relative magnitudes of the terms in the sum (1) are ultimately determined by the relative magnitudes of the associated quantities

$$
\alpha_{s k} \cos \tau+\beta_{s k} \sin \tau \text {. }
$$

These, however, are represented geometrically by the respective projection of the vectors $\bar{c}_{s k}$ upon the line (27), and from this interpretation it is quickly seen, in virtue of the restriction upon the range of $\tau$, that

$$
\alpha_{s k} \cos \tau+\beta_{s k} \sin \tau<\alpha_{r 1} \cos \tau+\beta_{r 1} \sin \tau, \text { for any } s \neq r .
$$

It follows that for $z$ on any line (27), that is, anywhere in the sector (28), the form of expression

$$
z^{\nu_{s k}} e^{c_{s} k^{z}}=z^{\nu_{r 1}} e^{c_{r 1} z} \boldsymbol{\epsilon}(z)
$$

is valid for $s \neq r$ and any $k$. The sum $\Phi(z)$ with coefficients of the form (16) may, therefore, be expressed as a sum with a reduced number of terms, that is,

$$
\Phi(z)=\sum_{h=1}^{h_{r}} A_{r h}(z) e^{c_{r h} z},
$$

and in this expression which is valid for all $z$ in the section (28) the coefficients are still of the form (16). The exponents in the sum (29) are, however, collinear, their conjugates $\bar{c}_{r h}$ lying on the side $l_{r}$. It may, therefore, be concluded from Theorem 7 that the zeros of $\Phi(z)$ within the sector (28) are asymptotically confined to one or more strips which are parallel or approach parallelism with the normal to the associated side of the polygon. A similar conclusion may obviously be drawn in connection with the values of $z$ in any other sector.

THEOREM 8. If in the sum (1) the exponents are any complex constants, the zeros of $\Phi(z)$ are confined for $|z|>M$ to a finite number of strips each of asymptotically constant width. These strips are associated in groups with the exterior normals to the sides of the polygon described in the text, and approach parallelism with the respective normals. Within each group of strips the dis- 
tribution of zeros may be described as in the previously stated theorems, the role of the axis of reals being transferred to the respective side of the polygon.

In closing here this brief and necessarily incomplete discussion of the distribution of the zeros of an exponential sum, I wish at least to call attention to the related work which has been done by $\mathrm{Ritt}^{8}$ on the factorization of such sums and on algebraic combinations of exponentials.

\section{Part II. The Exponential Integral}

10. Discussion of the Integral. If in the exponential sum with constant coefficients and real exponents as given in form (9), the origin is assumed to be a zero of the sum (and this can be made so by a simple translation of the plane), then $\sum_{j=0}^{n} a_{j}=0$. With this adjustment the sum $\Phi(z)$ can be simply represented in the form of an integral as follows. Let the coefficient function $\phi(t)$ be constant in successive intervals as defined by the relation

$$
\phi(t) \equiv-\sum_{j=0}^{r} a_{j}
$$

for $c_{r} \leqq t<c_{r+1},(r=0,1,2, \cdots, n-1)$. Then it is found directly that

$$
\Phi(z)=z \int_{c_{0}}^{c_{n}} \phi(t) e^{z t} d t .
$$

The exponential integral of the general type

$$
\Psi_{a, b}(z)=\int_{a}^{b} \psi(t) e^{z t} d t
$$

thus includes among its specializations (except for a factor $z$ ) the exponential sums of the types considered in $\$ 2$ and $\$ 3$, and hence, of course, also all trigonometric functions which are so expressible. Besides these a number of other functions, including Bessel functions, are amenable to representation in this form.

In turning to a consideration of the integral (30) a restriction of the discussion is necessitated by the brevity of available time. It is proposed, therefore, to summarize only certain investiga- 
tions which deal with the integral with finite limits of integration and which impose none but qualitative hypotheses on the coefficient function $\phi(t)$ involved. Many investigators have considered either integrals of specialized forms included under the type (30), or the integral with infinite limits. It is not due to a want of interest in the results obtained that these subjects are omitted from the present discussion.

Various normalizations of the integral (30) may be attained by simple substitutions on the variables involved. Appropriate normalizations of this kind make for simplicity in the enunciation of hypotheses and results, and for this reason the following ones will be assumed as indicated from point to point in the ensuing discussion. To begin with, the substitution of $\alpha t+\beta$ and $z / \alpha$, respectively, for $t$ and $z$ affects a change in the limits of integration, and by suitable choice of the constants $\alpha, \beta$, any desired finite limits may be attained. It will be found of particular convenience to normalize the interval thus in some cases to $(0,1)$ and in others to $(-1,1)$. Further, the substitution of $z+c$ for $z$ affects the introduction of a factor $e^{c t}$ into the coefficient function $\psi(t)$, and it follows that whenever $\psi(a) \neq 0$ and $\psi(b) \neq 0$, a normalization may be made such that $\psi(a)=\psi(b)=1$. Lastly it will be observed that the trigonometric in tegrals

$$
\begin{aligned}
& \Psi_{s}(z)=\int_{0}^{1} f(t) \sin z t d t \\
& \Psi_{c}(z)=\int_{0}^{1} f(t) \cos z t d t
\end{aligned}
$$

are both expressible in the form $\Psi_{-1,1}(i z)$, the coefficients being given respectively by the formulas $[1 /(2 i)]\{f(t)-f(-t)\}$, and $(1 / 2)\{f(t)+f(-t)\}$.

11. The Integral with an Infinity of Real Zeros. The distribution of zeros of an exponential sum (9) as determined in $\S 3$ features conspicuously the existence of a bound upon the numerical values of $x$ for which a zero of the sum may occur. This fact, it will be recalled, followed from the ultimate dominance of the one or the other extreme term of the sum when $|x|$ becomes sufficiently large. The integral (30) is in one sense the analogue of an exponential sum with infinitely many exponents lying on the range between $a$ and $b$. Either of these extreme 
values might in such event be a limit point for the infinite set, and if such were the case it would conceivably involve an indefinite delay in the accession to dominance of the respective extreme term of the sum. The absence of the corresponding bound upon the $x$ coordinate of the zeros might then be reasonably expected. That the situation thus conceived does as a matter of fact exist is shown by the following example constructed by Titchmarsh. ${ }^{9}$

Let the constants $\theta$ and $\nu$ be chosen subject to the conditions $\theta<1 / 2, \nu>2$, and let $\alpha=\nu / \theta$. The function

$\psi(t)=\left\{\begin{array}{cl}(-\alpha)^{k} e^{-\nu^{k}}, & \text { for } \theta^{k}-\alpha^{-k}<t \leqq \theta^{k} \quad(k=1,2,3, \cdots), \\ 0 & , \text { elsewhere on }(0,1),\end{array}\right.$

is integrable (absolutely) in the sense of Lebesgue. But for any positive integer $n$ it is found respectively that

$$
\begin{aligned}
\left|\int_{0}^{\theta^{n+1}} \psi(t) e^{-\alpha^{n} t} d t\right| \leqq \int_{0}^{\theta^{n+1}}|\psi(t)| d t \\
=\sum_{k=n+1}^{\infty} e^{-\nu^{k}}<e^{-\nu^{n+1}} \sum_{j=0}^{\infty} e^{-\nu^{j}}=e^{-\nu^{n+1}} \int_{0}^{1}|\psi(t)| d t
\end{aligned}
$$

that

$$
\begin{gathered}
\left|\int_{\theta^{n-1}-\alpha^{-n+1}}^{1} \psi(t) e^{-\alpha^{n} t} d t\right| \leqq e^{-\alpha^{n}\left(\theta^{n-1}-\alpha^{-n+1}\right)} \int_{0}^{1}|\psi(t)| d t \\
=e^{-\nu^{n} / \theta+\alpha} \int_{0}^{1}|\psi(t)| d t
\end{gathered}
$$

and that

$$
\int_{\theta^{n}-\alpha^{-n}}^{\theta^{n}} \quad \psi(t) e^{-\alpha^{n} t} d t=(-1)^{n}(e-1) e^{-2 \nu n}
$$

Hence it follows that

$$
\begin{aligned}
\mid e^{2 \nu^{n}} \Psi_{01}\left(-\alpha^{n}\right)-(e-1) & (-1)^{n} \mid \\
& <\left\{e^{\nu^{n}(2-\nu)}+e^{\nu^{n}(2-1 / \theta)+\alpha}\right\} \int_{0}^{1}|\psi(t)| d t,
\end{aligned}
$$


and since the quantity on the right approaches zero with $1 / n$ it must be concluded that $\Psi_{0,1}(z)$ takes when $z=-\alpha^{n}$ (and $n$ is sufficiently large) the sign of $(-1)^{n}$. The existence of at least one zero in each corresponding interval $-\alpha^{n+1} \leqq z \leqq-\alpha^{n}$ is thus established.

12. Hypotheses of Specific Type. While the example of the preceding section shows that the distribution of zeros of an exponential integral may differ sharply from that of an exponential sum, yet in a very wide class of cases they show considerable similarity. The coefficient function $\psi(t)$ is of course the determinative factor of the integral, and hence is always the direct subject of the hypothesis. The substance of the present section is essentially an abstract of results which are due to Pólya ${ }^{1}$ and is characterized by the fact that the hypotheses are on the whole of rather specific character. The results are accordingly precise to a considerable degree of detail.

If the function $\psi(t)$ is a step-function with discontinuities finite in number and occurring only at rational points of the interval, the function will be called exceptional. With such a function as coefficient the integral may be directly evaluated into the form of an exponential sum of the elementary type considered in \$2. Hence no essential loss is involved in the restriction of the theorems to unexceptional functions, and this will be tacitly assumed in the absence of statements to the contrary.

THEOREM 9. If the function $\psi(t)$ is positive and non-decreasing on the interval $(0,1)$, then the zeros of the integral $\Psi_{0,1}(z)$ are all located in the open half-plane $x<0$.

By way of proof let the value $z=i y$ be first considered. For any value $t_{1}$ on the interval of integration it is seen that

$$
\left|i y \int_{0}^{t_{1}} \psi(t) e^{i y t} d t\right| \geqq \psi\left(t_{1}\right)-\psi(0)-\left|\int_{0}^{t_{1}} e^{i y t} d \psi\right| .
$$

But if $\theta$ is defined by the relation

$$
\left|\int_{0}^{t_{1}} e^{i y t} d \psi\right|=e^{-i \theta} \int_{0}^{t_{1}} e^{i y t} d \psi
$$

then as $t_{1} \rightarrow 1$ the relation above becomes 


$$
\left|i y \Psi_{01}(i y)\right| \geqq \lim _{t_{1} \rightarrow 1} \int_{0}^{t_{1}}\{1-\cos (y t-\theta)\} d \psi .
$$

Since $d \psi$ is never negative, the conclusion $\left|\Psi_{01}(i y)\right|>0$ will be expected, and is in fact correct. Its derivation is, however, impeded somewhat by the necessity of recognizing and disposing of the potential effect of the functional dependence of $\theta$ upon the variable $t_{1}$. In conclusion the substitution of $z=x+i y$ in the discussion given merely replaces $\psi(t)$ by $\psi(t) e^{x t}$, and since this satisfies the hypotheses made for any fixed non-negative $x$, the assertion of the theorem is established.

The result obtained admits of ready extension into the following form.

THEOREM 10. If the coefficient $\psi(t)$ is positive and continuous, and if $\psi^{\prime}(t)$ exists (except possibly at a finite number of points) and

$$
\alpha \leqq-\frac{\psi^{\prime}(t)}{\psi(t)} \leqq \beta
$$

then the zeros of the integral $\Psi_{0,1}(z)$ are confined to the open strip of the complex plane given by the relation

$$
\alpha<x<\beta .
$$

(The trivial case $\psi(t)=e^{c t+d}$ is excepted.)

The transformations of the integral into the forms

$$
\Psi_{0,1}(z)=\int_{0}^{1} e^{\beta t} \psi(t) e^{(z-\beta) t} d t=e^{z} \int_{0}^{1} e^{-\alpha t} \psi(1-t) e^{(\alpha-z) t} d t
$$

are easily verified. In these forms the coefficients $e^{\beta t} \psi(t)$, and $e^{-\alpha t} \psi(1-t)$, satisfy the hypothesis of Theorem 9 , and hence the zeros are simultaneously restricted by the relations

$$
R(z-\beta)<0, R(\alpha-z)<0 .
$$

From this the theorem follows.

THEOREM 11. If the function $f(t)$ is positive and non-decreasing on the interval $(0,1)$, then the zeros of the trigonometric integrals $\Psi_{s}(z)$ and $\Psi_{c}(z)$ defined in (31) are all real and simple. The zeros of $\Psi_{c}(z)$ occur singly in each of the intervals

$$
[(m-1 / 2) \pi,(m+1 / 2) \pi], \quad m \neq 0,
$$


while those of $\Psi_{s}(z)$ occur (except for $z=0$ ) singly in each of the intervals $[m \pi,(m+1) \pi], m \neq 0,-1$.

The facts of this theorem may be established by suitable passages from finite to corresponding infinite sums as is indicated in the following. The trigonometric sum

$$
\Phi_{s}(z)=\frac{1}{n} \sum_{j=0}^{n} f\left(\frac{j}{n}\right) e^{j / n^{2}} \sin \left(\frac{j}{n} z\right)
$$

has, for each value of $n$, coefficients which are positive and increasing. Its zeros, therefore, by Theorem 2, are real, and alternate with those of the corresponding sum of cosines. From this it may be concluded, with suitable reasoning as $n \rightarrow \infty$, that the zeros of the integrals $\Psi_{s}(z)$ and $\Psi_{c}(z)$ are real, and that a multiple zero of the one would necessarily be also a zero of the other. Since in this latter event the integral

$$
\Psi_{c}(z)+i \Psi_{s}(z)=\int_{0}^{1} f(t) e^{i z t} d t
$$

would have a real zero, contrary to Theorem 9, the simplicity of the zeros must be granted.

The more precise location of the zeros as stated in the theorem is derived by Pólya from the expansion

(32) $\frac{\Psi_{s}(z)}{z \sin z}=\lim _{n \rightarrow \infty}\left\{\frac{\Psi_{s}{ }^{\prime}(0)}{z}-\sum_{j=1}^{n} \frac{(-1)^{j+1} \Psi_{s}(j \pi)}{j \pi}\left[\frac{1}{z-j \pi}+\frac{1}{z+j \pi}\right]\right\}$,

which may be established by methods familiar in the theory of functions of a complex variable. Each coefficient on the right of this expansion is positive, for

$$
(-1)^{j+1} \Psi_{s}(j \pi)=(-1)^{j+1} \int_{0}^{1} f(t) \sin j \pi t d t,
$$

while the integral represents a sum of areas, alternating in sign, non-decreasing in numerical magnitude, with the final one positive. Because of this the rational function within the brace in (32) is found by elementary means to have one zero in each of the intervals $(-\infty,-n \pi),(n \pi, \infty),[m \pi,(m+1) \pi], m=-n$, $\cdots,-1,1,2, \cdots, n-1$. A suitable passage to the limit as 
$n \rightarrow \infty$ establishes the theorem. An analogous discussion for $\Psi_{c}(z)$ is, of course, possible.

If the hypothesis of Theorem 11 is extended to include the assumptions that $\lim _{t \rightarrow 0} f(t)=0$, and that $f(t)$ is nowhere concave, a refinement of the theorem is possible which determines the specific half of each of the intervals given in which the zero is located. The method of proof is similar to that outlined above, a remark which applies also to the following theorem.

TheOREM 12. If the function $f(t)$ is positive, decreasing, and nowhere convex downward, and if $f^{\prime}(t)$ exists and is not an exceptional function, then the zeros of $\Psi_{c}(z)$ are all real and there is just one in each of the intervals $[m \pi,(m+1) \pi], m \neq 0,-1$.

As examples of integrals covered by the theorems of this section one has the familiar functions

$$
\begin{aligned}
& J_{0}(z)=\int_{0}^{1} \frac{2}{\pi\left(1-t^{2}\right)^{1 / 2}} \cos z t d t \\
& J_{1}(z)=\int_{0}^{1} \frac{2 t}{\pi\left(1-t^{2}\right)^{1 / 2}} \sin z t d t .
\end{aligned}
$$

13. Hypotheses of Intermediate Type. Less specific than those of the preceding section are the hypotheses and results to which the attention will now be turned. The material has been taken essentially from a memoir by Miss M. L. Cartwright ${ }^{10}$ under date of the present year. The two theorems to be given first, however, are attributed by her to Hardy.

As a preliminary it is perhaps desirable to note at this point the following relations which have been proved by Titchmarsh, ${ }^{9}$ namely that with any coefficient function $\psi(t)$ which is integrable,

$$
\left|\Psi_{a, b}(z)\right|\left\{\begin{array}{l}
=o\left(e^{|x|}\right), \\
\neq O\left(e^{|x|-|z| \delta}\right), \text { for any } \delta>0
\end{array}\right.
$$

These assertions may be established by a direct analysis of the integral involved.

Theorem 13. If the function $\psi(t)$ is itself an integral, if it is continuous at $t=1$ and $t=-1$, and if $\psi(1)=\psi(-1)=1$, then the 
zeros, $z_{m}$, of the integral $\Psi_{-1,1}(z)$ are given by the asymptotic formula

$$
z_{m} \sim m \pi i
$$

The proof is immediate. An integration by parts yields the equality

$$
z \Psi_{-1,1}(z)=e^{z}-e^{-z}-\int_{-1}^{1} \psi^{\prime}(t) e^{z t} d t .
$$

Of this the right hand member is of the form

$$
e^{z}\{1+\epsilon(z)\}-e^{-z}\{1+\epsilon(z)\},
$$

because of the first of the relations (33). That the zeros are located as stated in the theorem may be concluded then from the deductions of $\$ 2$ and $\$ 4$.

THEOREM 14. If the function $\psi(t)$ is of bounded variation, is continuous at $t=1$ and $t=-1$, and $\psi(1)=\psi(-1)=1$, then the zeros of $\Psi_{-1,1}(z)$ all lie within a strip of the complex plane given by a relation $|x|<K$.

The proof of this theorem has been constructed on the following lines. When $x>0$, an integration yields the evaluation

$$
z \Psi_{-1,1}(z)=e^{z}-e^{-z}-\int_{-1}^{1-\delta} e^{z t} d \psi-\int_{1-\delta}^{1} e^{z t} d \psi
$$

in which the integrals on the right are numerically less, respectively, than $H e^{x(1-\delta)}$, and $e^{x} \eta(\delta)$, with $H$ some constant and $\eta(\delta)$ the maximum of $\left|\psi\left(t_{2}\right)-\psi\left(t_{1}\right)\right|$ for $1-\delta<t_{1}<t_{2} \leqq 1$. Hence

$$
\left|z \Psi_{-1,1}(z)\right| \geqq e^{x}\left\{1-e^{-2 x}-H e^{-\delta x}-\eta(\delta)\right\} .
$$

However, the right member of this inequality is assuredly positive if $\delta$ is chosen sufficiently small (because of the continuity of $\psi(t)$ at $t=1$ this makes $\eta(\delta)$ small), and if $K$ is then chosen sufficiently large and $x>K$. It evidently follows that for such values of $z$ the integral can have no zero, and a similar discussion establishes the fact for $x<-K$.

The hypotheses of Theorem 14 are seen from the conclusions thus drawn to be sufficient to assure for the exponential integral a distribution of zeros resembling in at least one salient 
feature the distribution associated with an exponential sum. By the following theorem this similarity is shown to extend beyond the mere restriction of the zeros to a strip of the plane even to the density of zeros within that strip.

THEOREM 15. Under the hypotheses of the preceding theorem the number, $n(r)$, of zeros of the integral $\Psi_{-1,1}(z)$ which are in absolute value less than $r$ satisfies the relation

$$
n(r)=\frac{2 r}{\pi}+O(1) .
$$

It is readily found that this relation is included in (7) which restricts the zeros of exponential sums.

The proofs of Theorem 15, and of the following Theorem 16, are less simple than any which have hitherto been outlined. They are based upon facts and relations familiar in the theory of entire functions and involve considerable detail. The author of the theorem ${ }^{10}$ has deduced also a formula for the value of the quantity

$$
\int_{0}^{r} \frac{n(s)}{s} d s
$$

THEOREM 16. If the function $\psi(t)$ is continuous, with modulus of continuity $\omega(\delta)$ on the interval $(-1,1)$, and if $\psi(1)=\psi(-1)=1$, then the zeros of the integral $\Psi_{-1,1}(z)$ all lie within a region

$$
|x|<K|z| \omega\left(\frac{1}{|z|}\right)
$$

with a suitably chosen constant $K$, and

$$
n(r)=\frac{2 r}{\pi}+O\left(r \omega\left(\frac{1}{r}\right)\right) .
$$

It will be recalled that the modulus of continuity $\omega(\delta)$ is defined as the maximum value of $\left|\psi\left(t_{2}\right)-\psi\left(t_{1}\right)\right|$ for $t_{2}, t_{1}$ on $(-1,1)$ and $\left|t_{2}-t_{1}\right|<\delta$. As $\delta \rightarrow 0, \omega(\delta)=o(1)$, and in fact if the function satisfies a Lipschitz condition $\left|\psi\left(t_{2}\right)-\psi\left(t_{1}\right)\right|<A\left|t_{2}-t_{1}\right| \alpha$, 
$0<\alpha \leqq 1$, then $\omega(\delta)=O\left(\delta^{\alpha}\right)$. As in the case of the preceding theorem an evaluation of the quantity

$$
\int_{0}^{r} \frac{n(s)}{s} d s
$$

is also deducible. Moreover, it can be shown that the requirement of continuity over the entire interval $(-1,1)$ can, with suitable modification in the results, be replaced by hypotheses demanding continuity only near the end points.

14. The Integral with Most General Coefficient. The exponential integral of greatest generality results from the admission of any function which is absolutely integrable to the role of the coefficient $\psi(t)$. As will be indicated below even this minimum of hypotheses is not unproductive of significant results.

The function $\Psi_{0,1}(z)$ is analytic over the entire plane, and as such its zeros may be enumerated in the order of increasing absolute magnitude, thus, $z_{m}=r_{m} e^{i \theta_{m}}$. The following theorems involve a qualitative characterization of the density with which these zeros are distributed in the complex plane.

THEOREM 17. If the coefficient function $\psi(t)$ is absolutely integrable, the zeros $z_{m}$ of the integral $\Psi_{0,1}(z)$ are distributed so that the series

$$
\sum_{m=1}^{\infty} \frac{1}{\left|z_{m}\right|^{1+\epsilon}}
$$

converges for every $\epsilon>0$, and diverges for $\epsilon=0$.

As an entire function the integral $\Psi_{0,1}(z)$ is of order at most unity, as may be seen from the evident relation

$$
\left|\Psi_{0,1}(z)\right| \leqq e^{|z|} \int_{0}^{1}|\psi(t)| d t
$$

Inasmuch as this order at least equals the exponent of convergence, the statement of the theorem in so far as it concerns the convergence for $\epsilon>0$ is established. The divergence for $\epsilon=0$ is not so immediately verified. Independent proofs of it have, however, been given by both Pólya ${ }^{1}$ and Titchmarsh. ${ }^{9}$ The method employed by the latter may be indicated briefly as follows. 
Convergence of the series for $\epsilon=0$ would imply the absolute convergence of the canonical product

$$
P(z)=\Psi_{01}(0) \prod_{j=1}^{\infty}\left(1-\frac{z}{z_{j}}\right) .
$$

In such event, however, a product of this type is known to satisfy the relation

$$
|P(z)|=O\left(e^{|z| \eta}\right)
$$

with any positive constant $\eta$. Since $\Psi_{0,1}(z)$ is expressible in the form $\Psi_{0,1}(z)=e^{(\alpha+i \beta) z} P(z)$, its order being unity, it would follow that

$$
\left|\Psi_{0,1}( \pm x)\right|=O\left(e^{( \pm \alpha+\eta)|x|}\right)
$$

and this contradicts the second of the relations (33).

THEOREM 18. If the function $\psi(t)$ is absolutely integrable, then the zeros of the integral $\Psi_{0,1}(z)$ satisfy the relations

$$
\begin{gathered}
n(r) \sim \frac{r}{n}, \\
\sum_{m=1}^{\infty} \frac{\cos \theta_{m}}{\left|z_{m}\right|} \text { converges absolutely, } \\
\sum_{m=1}^{\infty} \frac{\sin \theta_{m}}{\left|z_{m}\right|} \text { converges conditionally. }
\end{gathered}
$$

These results have all been obtained by Titchmarsh $^{9}$ by the use of relations in the theory of entire functions. The result (a) evidently implies the assertions of Theorem 17, and is in fact more precise. The result (b) in conjunction with (a) indicates that in general $\left|\cos \theta_{m}\right|$ is small when $m$ is large. The example of $\$ 11$ would show, however, that a relation $\lim _{m \rightarrow \infty} \cos \theta_{m}=0$ is not in general valid. The result (c), which is obvious in the case of a real coefficient function $\psi(t)$, since the zeros present themselves then in conjugate pairs, is not easily established in the general case. The author of the result ${ }^{9}$ has, however, succeeded in explicitly summing both the series (b) and (c) in terms of the values taken by $\Psi_{0,1}(z)$ and its derivative at $z=0$. 


\section{LIST OF REFERENCES}

1. Pólya, G., Über die Nullstellen gewisser ganzer Funktionen, Mathematische Zeitschrift, vol. 2 (1918), pp. 352-383.

2. Wilder, C. E., Expansion problems of ordinary linear differential equations, etc., Transactions of this Society, vol. 18 (1917), pp. 415-442.

3. Tamarkin, J. D., Some general problems in the theory of ordinary linear differential equations, 1917, Petrograd; and Mathematische Zeitschrift, vol. 27 (1927), p. 24.

4. Birkhoff, G. D., Boundary value and expansion problems, etc., Transactions of this Society, vol. 9 (1908), p. 373.

5. Langer, R. E., The asymptotic location of the roots of a certain transcendental equation, Transactions of this Society, vol. 31 (1929), p.837.

6. Pólya, G., Geometrisches ïber die Verteilung der Nullstellen, etc., Sitzungsberichte der Bayerischen Akademie, München, 1920, p. 285.

7. Schwengler, E., Geometrisches über die Verteilung der Nullstellen, etc., Dissertation, Zürich, 1925.

8. Ritt, J. F., A factorization theory, etc., Transactions of this Society, vol. 29 (1927), p. 584.

On the zeros of exponential polynomials, and algebraic combinations of exponentials, Transactions of this Society, vol. 31 (1929).

9. Titchmarsh, E. C., The zeros of certain integral functions, Proceedings of the London Mathematical Society, vol. 25 (1926), p. 283.

10. Cartwright, M. L., The zeros of certain integral functions, Quarterly Journal of Mathematics, vol. 1 (1930), p. 38.

The University of Wisconsin 